\title{
Variabilidade da precipitação e sua relação com a produtividade agroindustrial do dendê no município do Acará, Estado do Pará, Brasil
}

Variability of separation and its relationship with the agro-industrial productivity of oil palm in the municipality of Acará, State of Pará, Brazil

Variabilidad de la separación y su relación con la productividad agroindustrial de la palma aceitera en el municipio de Acará, Estado de Pará, Brasil

Thays Valente do Nascimento ORCID: https://orcid.org/0000-0001-9797-2372 Universidade Federal do Pará, Brasil E-mail: thays.valente19@gmail.com Juliane Ribeiro das Chaves

ORCID: https://orcid.org/0000-0001-5141-1529 Universidade Federal do Pará, Brasil E-mail: juliane.rib21@gmail.com

Lilian Paixão Aleixo de Sousa ORCID: https://orcid.org/0000-0002-8673-1116 Universidade Federal do Pará, Brasil E-mail: lilianpaixao92@gmail.com

Francisco Carlos Lira Pessoa

ORCID: https://orcid.org/0000-0002-6496-9043 Universidade Federal do Pará, Brasil E-mail: fclpessoa@ufpa.br

Ana Beatriz Bastos Gomes

ORCID: https://orcid.org/0000-0003-0237-8210 Universidade Federal do Pará, Brasil E-mail: abeatrizbgomes@gmail.com

Bruna Roberta Pereira Lira

ORCID: https://orcid.org/0000-0003-4152-7709 Universidade Federal do Pará, Brasil E-mail: bruna.lira@itec.ufpa.br

Dayana Cravo Rodrigues

ORCID: https://orcid.org/0000-0002-5876-6901

Universidade Federal do Pará, Brasil

E-mail: dayana_cravo@ hotmail.com

Lucilla Raphaelle Carmo Castro

ORCID: https://orcid.org/0000-0003-2284-5912 Instituto Federal de Educação, Ciência e Tecnologia do Pará, Brasil E-mail: leluci.castro@gmail.com

Luiza de Nazaré Almeida Lopes ORCID: https://orcid.org/0000-0001-7988-9313 Instituto Federal de Educação, Ciência e Tecnologia do Pará, Brasil E-mail: luiza.lopes@ifpa.edu.br

\begin{abstract}
Resumo
O Brasil é um país no qual a produtividade agrícola é das principais atividades que contribui para o PIB do país. A região amazônica é conhecida por sua abundância de água e por ter uma alta pluviosidade, este é um dos fatores que contribuem para agricultura. Neste aspecto, o objetivo deste trabalho foi analisar a correlação entre a variabilidade pluviométrica e a produtividade agrícola no município de Acará - Pará, e assim, verificar a influência da precipitação na produção de dendê. Foram utilizadas séries pluviométricas fornecidas no Sistema de Informações Hidrológicas (HidroWeb) e dados de produtividade no banco de dados do Sistema IBGE de Recuperação Automática - SIDRA, nas séries de chuva aplicou-se teste estatístico não paramétrico de Mann-Kendall. Por meio do método de correlação linear, correlacionou-se variáveis de total anual de precipitação e produtividade anual agrícola. A partir da análise dos dados, verificou-se uma correlação em torno de $40 \%$ entre o cultivo da cultura de dendê e a precipitação, um valor considerado moderado, logo as chuvas são consideradas importantes, mas não um fator limitante para o cultivo deste fruto.
\end{abstract}


Palavras-chave: Precipitação; Produtividade; Correlação; Dendê.

\begin{abstract}
Brazil is a country without agricultural productivity qualifications and is one of the main activities that contribute to the country's GDP. The Amazon region is known for its abundance of water and high rainfall, this is one of the factors that contribute to agriculture. In this respect, the objective of this work was to analyze a correlation between rainfall variability and agricultural productivity in the city of Acará - Pará, and, thus, to verify the influence of capacity in oil palm production. Rainfall series, provided in the Hydrological Information System (HidroWeb) and productivity data without a database from the IBGE Automatic Recovery System - SIDRA, were used in the rain series, Mann-Kendall non-parametric statistical test was applied. Through the linear correlation method, the variables of annual total capacity and annual agricultural productivity were correlated. From the data analysis, there was a correlation around $40 \%$ between the cultivation of palm oil and a separation, a value considered moderate, as rains are considered important, but not a limiting factor for the cultivation of this fruit.
\end{abstract}

Keywords: Precipitation; Productivity; Correlation; Palm oil.

\title{
Resumen
}

Brasil es un país en el que la productividad agrícola es una actividad importante que contribuye al PIB del país. La región amazónica es conocida por su abundancia de agua y por tener una alta precipitación, este es uno de los factores que contribuyen a la agricultura. Al respecto, el objetivo de este trabajo fue analizar la correlación entre la variabilidad de las precipitaciones y la productividad agrícola en la ciudad de Acará - Pará, y así, verificar la influencia de la precipitación en la producción de aceite de palma. Se utilizaron series de precipitaciones proporcionadas en el Sistema de Información Hidrológica (HidroWeb) y datos de productividad en la base de datos del Sistema de Recuperación Automática IBGE - SIDRA, en la serie de lluvias se aplicó la prueba estadística no paramétrica de Mann-Kendall. Mediante el método de correlación lineal se correlacionaron las variables de precipitación total anual y productividad agrícola anual. Del análisis de los datos se encontró una correlación de alrededor del $40 \%$ entre el cultivo de aceite de palma y la precipitación, valor considerado moderado, por lo que las lluvias se consideran importantes, pero no un factor limitante para el cultivo de este fruto.

Palabras clave: Precipitación; Productividad; Correlación; Aceite de palma.

\section{Introdução}

O Brasil é um dos grandes produtores agrícolas do mundo, por apresentar condições favoráveis ao desenvolvimento das mais diversas culturas, como por exemplo as áreas extensas, variabilidade do clima e solos propícios à plantios. A agricultura é uma das atividades que mais contribui para o PIB (Produto Interno Bruto). Segundo dados estatísticos do IBGE (Instituto Brasileiro de Geografia e Estatística), no ano de 2017 o PIB teve um crescimento acumulado da agropecuária de $14,5 \%$, resultado principalmente da agricultura (IBGE, 2017).

A agricultura não é apenas responsável por grande parte das exportações brasileiras e pela geração de milhares de empregos, mas também uma das atividades mais vulneráveis às mudanças climáticas, e áreas atualmente recomendadas para a produção de culturas podem ser mudadas (Silva, Prela-Pantano, \& Sant'anna Neto, 2008; Lima \& Alves, 2008). Essa produção agrícola depende, principalmente, do solo e das condições climáticas, tais como, temperatura e precipitação, pois atuam no desenvolvimento das plantas (Silva et al., 2008). O regime pluviométrico é o que exerce maior influência sobre as condições ambientais dentre os elementos meteorológicos (Dallacort, Martins, Inoue, Freitas, \& Coletti, 2011). Dessa forma, conforme Silva et al. (2008), a organização do espaço agrícola deveria partir de uma concepção dos atributos climáticos, não como determinantes, mas como insumo nos processos naturais e de produção.

O consumo de água pela vegetação é uma informação relevante no planejamento agrícola, com vistas à avaliação da produtividade das culturas (Campos et al., 2008). As necessidades hídricas das culturas agrícolas precisam ser satisfeitas nos diversos estágios, desde a germinação e estabelecimento da cultura, passando pelo desenvolvimento, floração, formação e crescimento dos grãos ou frutos, até atingir a maturação e a colheita (Silva et al., 2008). A partir desse conhecimento, é possível gerar informações e ferramentas mais precisas em escala regional e global. Podem-se elaborar e implementar políticas públicas para redução dessas vulnerabilidades e aumento da capacidade adaptativa da população, da economia e, na medida do possível, dos ecossistemas (Nobre, 2008; Minuzzi, 2010). 
No cultivo agrícola é imprescindível se conhecer as relações entre as chuvas da pré-estação e período chuvoso para escolha da melhor época de semeadura, devido às condições hídricas do solo (Silva et al., 2008). Assim, verifica-se que a região é importante para produção agrícola e essa produção é muito interligada com o regime pluviométrico, por isso é necessário saber, além do total de chuva, a variabilidade e as probabilidades da ocorrência da precipitação na região para realizar um bom planejamento para as atividades agrícolas. Segundo Carmello e Sant'Anna Neto (2015), mesmo com investimentos que minimizem os efeitos de variabilidade de chuvas, a produção agrícola depende significativamente do tempo e do clima. Os fatores tempo e clima correspondem de $60 \%$ a $70 \%$ da dificuldade que o ser humano tem para contornar em relação às atividades agrícolas (Carmello \& Sant'Anna Neto, 2015).

O dendê (Elaeis guineenses) é conhecido internacionalmente como palma-africana e no Brasil como dendezeiro. Sua produção caracteriza-se como uma atividade agroindustrial, com consideráveis investimentos em infraestrutura social e utilização intensiva de mão de obra, sem problemas de entressafras e com geração de empregos de boa qualidade, pela alta rentabilidade da cultura (Silva, 2006). As chuvas para o cultivo de dendê devem ser bem distribuídas no decorrer do ano, sem a ocorrência de estações secas definidas, com média de $2000 \mathrm{~mm}$. Nos meses chuvosos, a precipitação pluviométrica não deve, preferencialmente, ser inferior a $1000 \mathrm{~mm}$ e nem superior a três meses (Barreto, 2012).

$\mathrm{O}$ dendê adapta-se a áreas desmatadas e solos degradados e apresenta alta produtividade nas condições amazônicas. (Cardoso, Maneschy, \& Matlaba, 2014). No Pará, a produtiva do dendê (Elaeis guineensis), cultura destinada à produção de óleo, vem sendo estabelecida desde a década de 1980 e com maior estímulo, a partir dos últimos anos. O Nordeste Paraense é a região mais próspera para a expansão do plantio de dendê conforme indicam pesquisas realizadas por instituições como a Embrapa, além das ótimas condições edafoclimáticas. Essa região é dotada de localização estratégica com infraestrutura de transporte, energia, comunicação, disponibilidade de mão de obra e adjacente aos mercados usuários, especialmente o externo. (Silva Junior, 2017).

Na região amazônica o clima é quente e úmido, com alto índice pluviométrico, com uma umidade relativa do ar anual de $80 \%$ a $81 \%$ (PTDRS, 2010). Moraes, Costa, Costa e Costa (2005) afirmam que a precipitação é reconhecida como a variável climatológica principal na região tropical. A elevada precipitação é um fator limitante ao desenvolvimento e à estabilização da produção agrícola no estado do Pará (SAGRI, 1996). Consequentemente, a variabilidade das chuvas em períodos de colheita agrícola geralmente determina a produção agrícola final. (Carmello \& Sant’Anna Neto, 2015).

$\mathrm{O}$ estudo da variabilidade de precipitação em uma região onde a agricultura possui representatividade é justificada pela necessidade de se compreender os fenômenos atmosféricos com relação a produção agrícola. Desta forma, o objetivo desse estudo foi analisar a correlação entre a variabilidade pluviométrica e a produtividade de dendê no município de Acará PA. Para isso, levou-se em consideração o regime pluviométrico como insumo essencial para produção desta cultura. A avaliação foi feita para o período de 1990 a 2014.

\section{Metodologia}

\section{1 Área de estudo}

O município de Acará, localizado no estado do Pará, situa-se à margem esquerda do rio Acará, na zona fisiográfica Guajarina. A exploração do território ocorreu no período de colonização da Província do Grão Pará. Segundo tradição, o rio Acará foi um dos primeiros cursos d'água a receber exploradores portugueses e colonos (IBGE, s/d). Acará pertence à Mesorregião Nordeste Paraense e à Microrregião Homogênea de Tomé-Açu (FAPESPA, 2016). O município possui uma extensão territorial de 4.344,384 km² e uma população estimada para o ano de 2020 de 55.669 pessoas (IBGE, 2020). Na Figura 1, está a localização do município do Acará e a estação pluviométrica selecionada para este estudo. 
Figura 1 - Localização do município do Acará, estado do Pará.

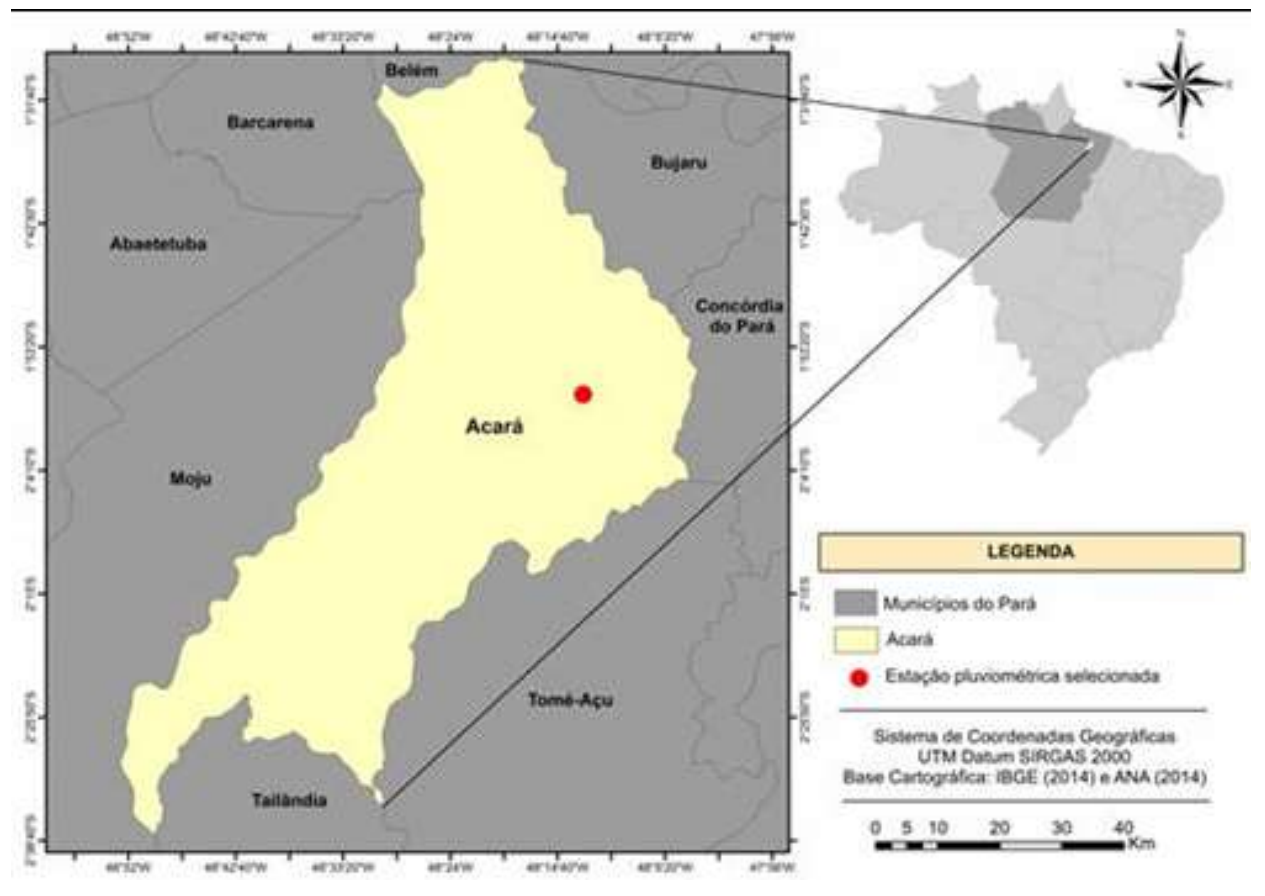

Fonte: Autores (2020)

Os solos da região são do tipo Latossolo Amarelo, textura argilosa e média, e Concrecionários Lateríticos, em áreas de terra firme. Diferentemente, em outras áreas são comuns os solos de várzeas, destacando-se os solos Hidromórficos indiscriminados eutróficos e distróficos, textura indiscriminada (FAPESPA, 2016). Quanto à topografia, o município não apresenta altitudes expressivas. Na sede municipal a cota média é de 35 metros e a nordeste desta sede é de 85 metros. O terreno predominante em Acará é a Formação Barreiras, e o relevo é pouco expressivo: na porção sul, dominam áreas de tabuleiros e colinas baixas aplainadas, características do relevo de terras firmes da Formação Barreiras (FAPESPA, 2016).

A região do município do Acará está inserida na bacia hidrográfica do rio Acará. Esta bacia é favorecida pela sua posição geográfica, com grande disponibilidade de águas superficiais e subterrâneas. E é caracterizada por uma série de canais (Silva Júnior, 2017).

O município de Acará não possui estação meteorológica, por isso são considerados dados do município próximo: Belém, logo, considera-se o município na faixa equatorial. O clima tipo Af da classificação de Köppen, correspondente ao tropical úmido. A temperatura mensal é superior a $18^{\circ} \mathrm{C}$, com chuvas nas duas estações, sendo a precipitação sempre acima de $60 \mathrm{~mm}$, em relação às aferições mensais. As precipitações anuais são em média de 2.837 mm e a amplitude térmica anual em torno de $5^{\circ} \mathrm{C}$ (FAPESPA, 2016).

\subsection{Levantamento de dados}

Para a realização desta pesquisa foram utilizados os dados de chuva, disponíveis no Sistema de Informações Hidrológicas (HidroWeb) da Agência Nacional de Águas (ANA). A descrição da estação pluviométrica escolhida, especificamente 148009 - Acará, está na Tabela 1. Esta estação foi selecionada por apresentar um banco de dados com mais de 30 anos de observação e poucas falhas. Assim, foi determinado o período de estudo de 1990 a 2014. 
Tabela 1 - Descrição da estação do Acará.

\begin{tabular}{cccccccc}
\hline CÓDIGO & ESTAÇÃO & MUNICÍPIO & $\begin{array}{c}\text { ÓRGÃO } \\
\text { OPERADOR }\end{array}$ & $\begin{array}{c}\text { LAT. } \\
\left({ }^{\circ}\right)\end{array}$ & $\begin{array}{c}\text { LONG. } \\
\left({ }^{\circ}\right)\end{array}$ & $\begin{array}{c}\text { ALT. } \\
(\mathbf{m})\end{array}$ & PERÍODO \\
\hline 148009 & Acará & Acará & CPRM & $1^{\circ} 57^{\prime} 40^{\prime \prime}$ & $48^{\circ} 12^{\prime} 23^{\prime \prime}$ & 0 & $1990-2014$ \\
\hline
\end{tabular}

Fonte: Autores.

Inicialmente foi realizado um estudo preliminar dos dados da estação, com o preenchimento das falhas e da verificação da consistência dos mesmos. A partir disso, foram obtidas as séries da precipitação média mensal e total anual para analisar a variabilidade, tendência pluviométrica e a correlação desta variável com a produtividade de dendê. Os dados de produção de Dendê do município do Acará foram obtidos no banco de dados da Produção Agrícola Municipal (PAM) do Sistema IBGE de Recuperação Automática - SIDRA, que possui as seguintes informações: Área plantada (ha), área colhida (ha), quantidade produzida ( $\mathrm{t}$ ) e produtividade média ( $\mathrm{kg} / \mathrm{ha}$ ), no período que compreende os anos de 1990 a 2014. Essa cultura foi escolhida por se tratar de uma das mais importantes para o desenvolvimento econômico regional.

\subsection{Análise estatística de tendência}

O teste de Mann-Kendall é um teste não paramétrico sugerido pela Organização Meteorológia Mundial (OMM) para avaliação da tendência em séries temporais de dados ambientais. $O$ teste assume a hipótese que a sequência de dados da série hidrológica ocorre de forma independente e a distribuição de probabilidade deve permanecer sempre a mesma (Sena \& Lucena, 2013; Yue, Pilon, \& Cavadias, 2002). Cada valor da série histórica analisada é comparada com os dados restantes em ordem sequencial, e são contabilizados o número de vezes que os valores restantes são superiores ao valor analisado. O teste é baseado na estatística S definida como (Onyutha, Tabari, Taye, Nyandwaro, \& Willems, 2015; Ribeiro, Ávila, Brito, Santos, \& Sousa, 2014; Sanches, May, Silva, \& Ferreira, 2016; Silva, Teixeira-Gandra, Damé, Klumb, \& Veber, 2015):

$$
S=\sum_{i=2}^{n} \sum_{j=1}^{i-1} \operatorname{sgn}\left(x_{i}-x_{j}\right)
$$

Em que 'xj' são os valores de dados sequenciais, 'n' é o comprimento da série temporal e 'sgn(xi-xj)' é igual a -1 para ' $\operatorname{sgn}(x i-x j)<0$ ', 0 para ' $\operatorname{sgn}(x i-x j)=0$ ', e 1 para ' $\operatorname{sgn}(x i-x j)>0$ '.

Para valores n grandes, sob a hipótese nula (H0) de ausência de tendência, S apresentará uma distribuição normal com média e variância dada, respectivamente, pelas seguintes equações:

$$
\begin{gathered}
E(S)=0 \\
\operatorname{Var}(\mathrm{S})=\frac{\mathrm{n}(\mathrm{n}-1)(2 \mathrm{n}+5)}{18}
\end{gathered}
$$

A estatística do teste $Z$ é dada pela equação:

$$
Z=\quad \begin{array}{cc}
\frac{S-1}{\sqrt{\operatorname{Var}(S)}} & \text { se } S>0 \\
0 & \text { se } S=0
\end{array}
$$




$$
\frac{S+1}{\sqrt{\operatorname{Var}(S)}} \text { se } S<0
$$

A presença de uma tendência estatisticamente significativa é realizada com base na análise da estatística $\mathrm{Z}$ e com isso é possível aceitar ou rejeitar H0, ou seja, é possível afirmar a existência ou não de tendência. Assim, H0, é rejeitada sempre que $|Z|>Z \alpha / 2$, em que $Z \alpha / 2$ é adotado da tabela de distribuição normal padronizada, que para o nível de significância adotado neste estudo é de $Z \alpha / 2$ =1,96 (Lima, 2014; Ribeiro et al., 2014; Yue et al., 2002). Assim, para um teste bilateral, a H0 será rejeitada se o valor de $Z$ estiver fora do intervalo de confiança $(-1,96 /+1,96)$ e será aceita caso o valor de $Z$ esteja dentro deste intervalo. $\mathrm{O}$ sinal da estatística $\mathrm{Z}$ indicará se a tendência é positiva $(\mathrm{Z}>0)$ ou negativa $(\mathrm{Z}<0)$. Dessa forma, as séries do total anual de precipitação e de produção anual de dendê, considerando um nível de significância de $5 \%$, foram submetidas ao teste estatístico de Mann-Kendall, através do software BioEstat, versão 5.0, para verificar a existência de possíveis tendências e a significância estatística das mesmas.

\subsection{Correlação entre precipitação e produtividade de dendê}

Foi analisada a correlação entre a precipitação total anual com as variáveis de produtividade total anual de dendê, através do método de correlação linear para obtenção do coeficiente de correlação (r). A análise foi feita por meio do software BioEstat, versão 5.0, comprovando se existe realmente a influência da precipitação na produtividade de dendê. Os valores de correlação entre as variáveis, podem mostrar vários níveis de correlação, desde de bem fraca até muito forte. A classificação utilizada para a interpretação de valores é: correlação bem fraca $(0$ a 0,19$)$, correlação fraca $(0,20$ a 0,39$)$, correlação moderada $(0,40$ a 0,69$)$, correlação forte $(0,70$ a 0,89$)$ e correlação muito forte $(0,90$ a 1,00) (Carvalho, et al., 2019).

\section{Resultados e Discussão}

\subsection{Variabilidade da precipitação média anual e sazonal}

A precipitação média mensal do município de Acará no período de 1990 a 2014 está ilustrada na Figura 2. Pode-se notar que existem dois períodos bem definidos: um período chuvoso compreendendo os meses de janeiro a junho; e um período menos chuvoso que compreende os meses de julho a dezembro. Os meses de março e abril foram os que apresentaram o maior índice pluviométrico, enquanto que os meses de setembro e outubro o menor índice. 
Figura 2 - Precipitação média mensal do município do Acará/ PA (1990-2014).

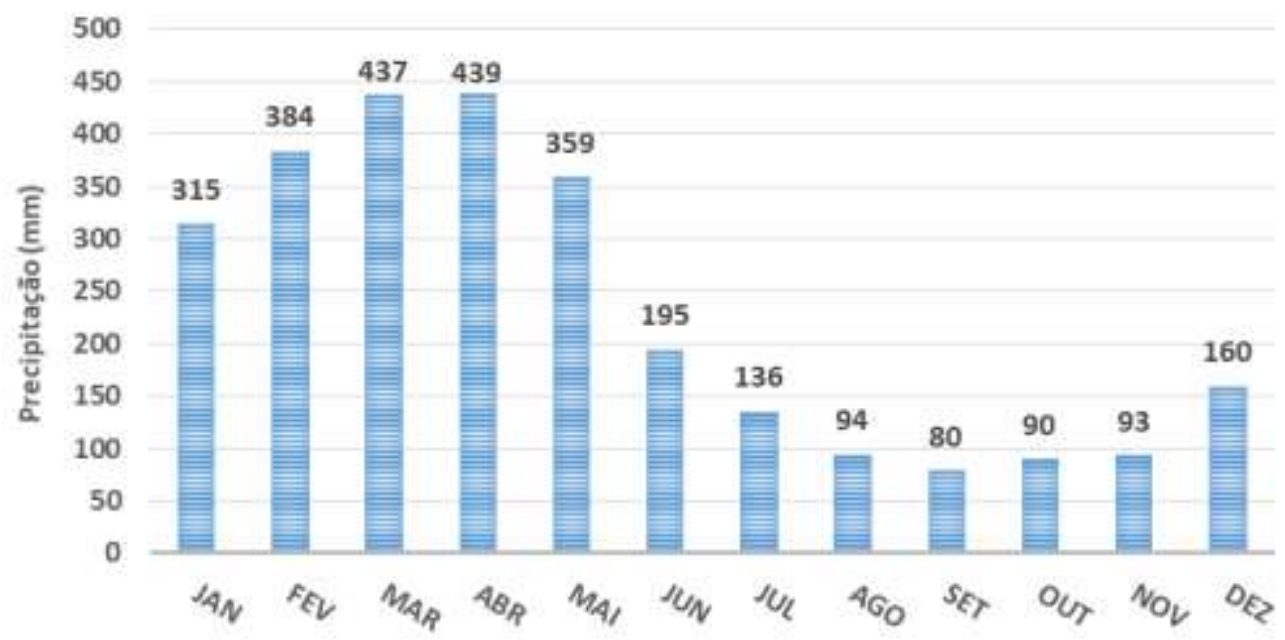

Fonte: Autores (2020).

Na Figura 3 é apresentada a precipitação total anual do município. Percebe-se que existe uma grande variabilidade interanual pluviométrica na região. A média da precipitação total anual foi $2.782 \mathrm{~mm}$, e apresentou uma variação de 1.663 mm no ano de 1990 a 4.893 mm no ano de 2009. Em comparação a outros estudos realizados em Belém do Pará, observa-se na pesquisa dos autores Moura \& Vitorino (2012), que a precipitação em Belém no período de 1999 a 2008 esteve acima de 3000 mm em seis dos dez anos analisados, assim como observa-se de forma semelhante nesta pesquisa, especificamente nos anos de: 1994, 2000, 2007, 2009 e 2014.

Figura 3 - Precipitação total anual do município do Acará/ PA (1990-2014).

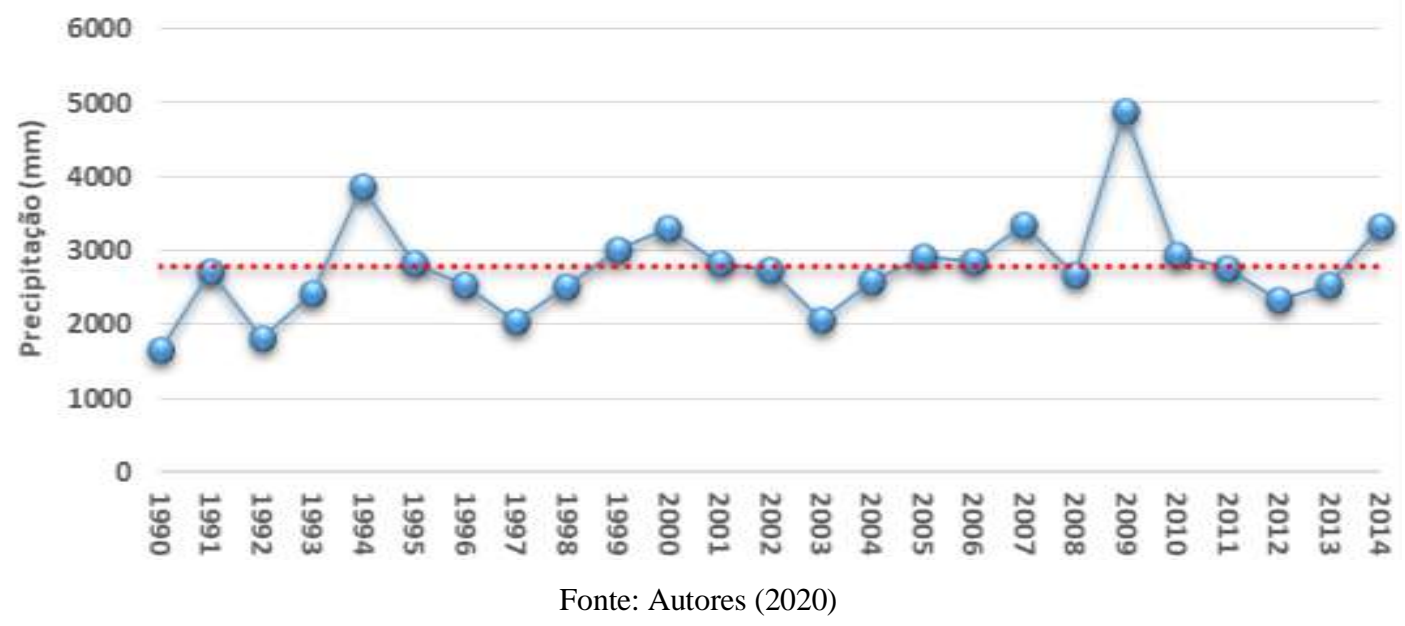

A partir da análise da série da estação do Acará, verificou-se a ocorrência de baixos índices pluviométricos nos anos de 1990 (1663 mm) e 1992 (1808 mm), que podem ter sido ocasionados pela ocorrência de El Niño nesses anos, de acordo com INPE/CPTEC (2016). Apesar da região em estudo se localizar próxima à linha do Equador, e ter abundância hídrica e solar, fatores que favorecem as precipitações, além desses fatores há outros que influenciam na pluviometria regional. De acordo com outros estudos a variabilidade pluviométrica é consequência de sistemas atmosféricos que atuam no estado do Pará (Lira, Lopes, Chaves, Santana, \& Fernandes, 2020; Almeida, Oliveira Jr., Delgado, Cubo, \& Ramos, 2017; Oliveira, Silva, \& Lima, 2017). Os sistemas atmosféricos de maior relevância para a incidência de chuva são: Zona de Convergência Intertropical 
(ZCIT), brisa marítima e as linhas de instabilidades (LI). Além dos fenômenos climáticos tropicais El Niño e La Niña (Lira et al., 2020; Moreira, Ferreira, Dias, \& Vitorino, 2019; Moraes \& Francisco Filho, 2018; Souza et al., 2017; Lopes, Souza, \& Ferreira, 2013; Campos, Mota, \& Santos, 2015).

\subsection{Tendências pluviométricas e na produtividade de dendê}

Para o nível de significância de 5\% e comparando o valor de $\mathrm{Z}$ do teste de Mann-Kendall com o intervalo de confiança $(-1,96$ a $+1,96)$, analisou-se a existência de tendências significativas no total anual da série pluviométrica do Acará, assim como na série de produtividade de Dendê.

Os resultados obtidos com a aplicação do Teste de Mann-Kendall indicaram que o índice da precipitação total anual apresenta uma tendência positiva, cerca de 1,82 mm/ano, no entanto, essa tendência não apresentou significância estatística, ou seja, a hipótese nula de que não há tendência foi aceita.

A produtividade de dendê apresentou uma forte tendência de aumento no período estudado, de cerca de 5,49 t/ano, sendo esta tendência significativa ao nível de 5\%. Os resultados obtidos com a aplicação do método estatístico são apresentados na Tabela 2 e podem ser visualizados nas Figuras 4 e 5.

Tabela 2 - Resultado do teste estatístico de Mann Kendall.

\begin{tabular}{ccc}
\hline \multirow{2}{*}{ Variável } & $\mathbf{Z}$ & Mann-Kendall \\
\cline { 2 - 3 } & $+1,8217$ & Significância Estatística \\
\hline Precipitação & $+5,4887$ & $\mathrm{~S}$ \\
\hline Produtividade & \multicolumn{2}{c}{ N }
\end{tabular}

Figura 4 - Tendência pluviométrica anual no município do Acará/ PA (1990-2014).

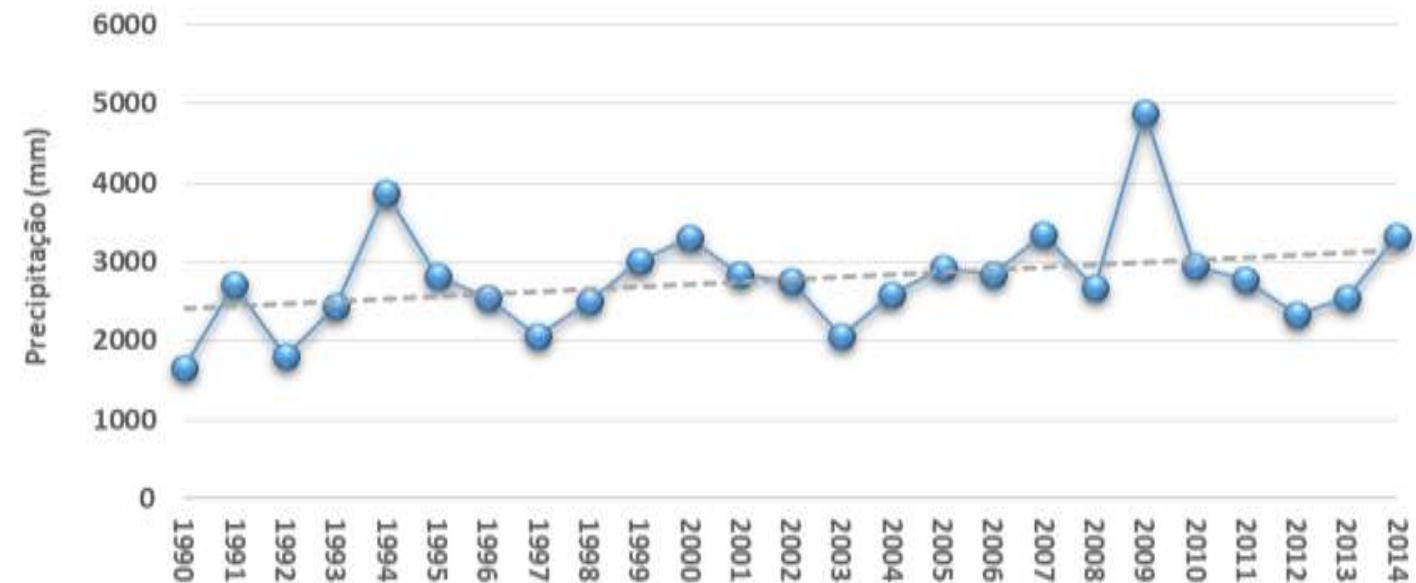

Fonte: Autores (2020). 
Figura 5 - Tendência na produtividade anual de dendê no município do Acará/ PA (1990-2014).

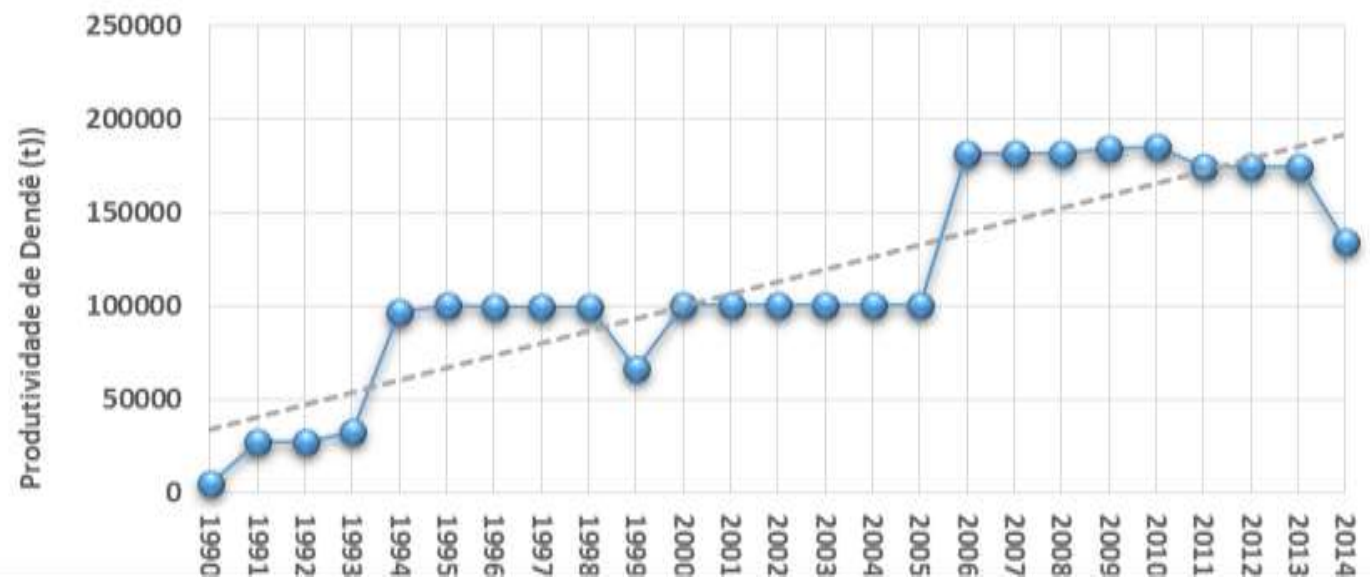

Fonte: Autores (2020)

As tendências de incremento apresentadas nos dois parâmetros, evidencia condições pluviométricas propícias para o desenvolvimento da cultura de dendê na região estudada, uma vez que, de acordo com Barcelos, Pacheco, Müller, Viégas e Tinôco (1987), a precipitação é o elemento climático mais importante para o crescimento e a produção do dendezeiro, em razão do dendê ser muito exigente em água, não tolerando regiões secas.

\subsection{Correlação entre precipitação e produtividade de dendê}

No período analisado, todas as variáveis de produção anual de dendê apresentaram correlações moderadas com os dados de precipitação em torno de 0,4 , ou seja, a precipitação influencia aproximadamente apenas $40 \%$ da produção de dendê no município do Acará, conforme dados apresentados na Tabela 3. Isso indica que outros fatores, como solo, temperatura, insolação, umidade, entre outros fatores, podem ser mais limitantes do que a pluviometria da região.

De acordo com Simas e Pereira (2019); Silva, Homma e Pena (2011), o dendezeiro requer temperatura média entre 25 e $27^{\circ} \mathrm{C}$, precipitações mensais mínimas de $100 \mathrm{~mm}$, umidade do ar próxima de $80 \%$ e uma luminosidade de pelo menos 5 horas de luz solar, por dia. Autores como Silva, Homma e Pena (2011) e Silva Junior (2017), enfatizam ainda, que a insolação é um fator muito importante para a cultura do dendê, pois esta implica de forma direta na produção, visto que a planta apresenta, elevada capacidade fotossintética, exigindo cerca de 1.500 a 2.000 horas anuais de luminosidade solar, com homogeneidade de distribuição ao longo do ano. Para Barcelos et al. (1987), o dendê tem melhor desenvolvimento em solos profundos, permeáveis, sem obstáculos para o desenvolvimento das raízes e pH entre 4,5 e 6,0.

Tabela 3 - Correlação Precipitação x Produtividade.

\begin{tabular}{l|c}
\hline \multicolumn{1}{c}{ Correlação das Variáveis de Produção } \\
\hline \multicolumn{1}{c}{ Variáveis } & Coeficiente de Correlação (r) \\
\hline Quantidade de cachos de dendê $(\mathrm{t})$ & 0,4533 \\
\hline Área colhida (ha) & 0,4348 \\
\hline Área plantada (ha) & 0,4260 \\
\hline Rendimento (Kg/ha) & 0,4162 \\
\hline
\end{tabular}

Fonte: Autores (2020)

Na Figura 6, pode ser visualizado essa relação, apesar de haver alguns anos, como os anos de 1990 a 1994, em que a produtividade sofre influência com a precipitação, nos demais anos nota-se que não há forte relação entre as duas variáveis. Isto corrobora que existem outros fatores que podem ser mais limitantes para a cultura do dendê que a precipitação. 
Figura 6 - Relação Precipitação x Produtividade de dendê (1990-2014).

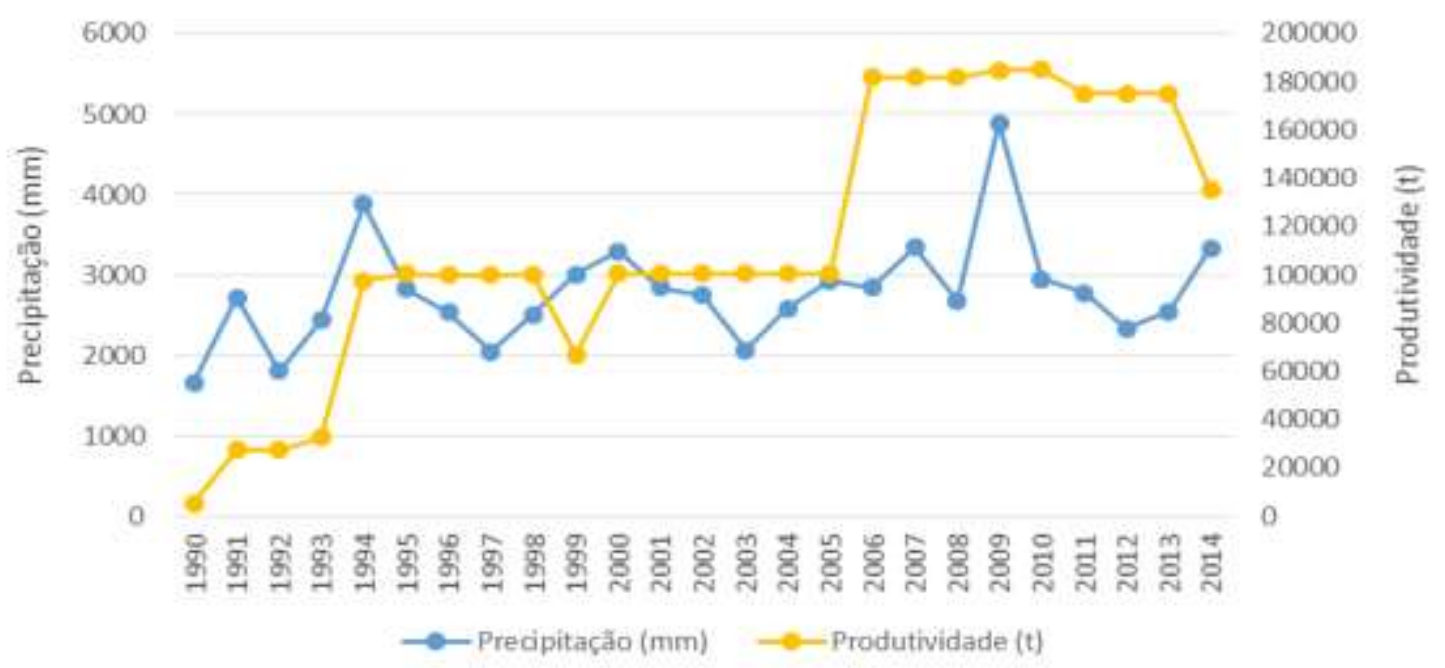

Fonte: Autores (2020).

\section{Considerações Finais}

A partir da análise dos dados, verificou-se uma correlação em torno de $40 \%$ entre o cultivo da cultura de dendê e a precipitação, um valor considerado moderado, logo as chuvas são consideradas importantes, mas não um fator limitante para o cultivo deste fruto na região estudada.

Além da precipitação outros aspectos devem ser correlacionados com produtividade de dendê, como por exemplo, a temperatura, qualidade do solo, tecnologia utilizada e mão de obra.

Sugere-se para trabalhos futuros a análise da correlação pluviométrica com a produtividade de dendê, levando em consideração a sazonalidade da região nos períodos mais chuvosos e menos chuvosos.

\section{Referências}

Agência Nacional de Águas. (2016). Sistema de Informações Hidrológicas (HidroWeb). http://hidroweb.ana.gov.br.

Almeida, C. T., Oliveira Jr., J. F., Delgado, R. C., Cubo, P. \& Ramos, M. C. (2017). Spatiotemporal rainfall and temperature trends throughout the Brazilian Legal Amazon. International Journal of Climatology, 37(4), 2013-2026.

Barcelos, E., Pacheco, A. R., Müller, A. A., Viégas, I. J. M., \& Tinôco, P. B. (1987). Dendê: Informações básicas para o seu cultivo, EMBRAPA-UEPAE de Belém. Documentos.Barreto,

A. G. T. (2012). Vulnerabilidade de agricultores familiares da cadeia de produção de biodiesel de dendê à extremos de precipitação na comunidade Águas Pretas, Moju (PA). Dissertação de Mestrado, Universidade Federal do Pará, Belém, PA, Brasil.

Campos, J. H. B. da C., Silva, V. de P. R., Azevedo, P. V., Borges, C. J. R., Soares, J. M., Moura, M. S. B., Silva, B. B. (2008). Evapotranspiração e produtividade da mangueira sob diferentes tratamentos de irrigação. Revista Brasileira de Engenharia Agrícola e Ambiental, 12 (2), $150-156$.

Campos, T. L. O. B., Mota, M. A. S. \& Santos, S. R. Q. (2015). Eventos extremos de precipitação em Belém-PA: uma revisão de notícias históricas de jornais. Revista Ambiente e Água, 10(1), 182-194.

Cardoso, A. C. D., Maneschy, M. C., Matlaba, V. J. (2014). Produção de óleo de palma no nordeste do Estado do Pará, Brasil: Desafios e subsídios para o desenvolvimento sustentável. Estudos Sociedade e Agricultura, 22(1), 193-223.

Carmello, V., \& Sant'Anna Neto, J. L. (2015). Variabilidade das chuvas na vertente paranaense da bacia do rio Paranapanema - 1999-2000 a 2009-2010. Raega - O Espaço Geográfico em Análise, 33, 225-247.

Carvalho, M. S., Andreozzi, V. L., Codeço, C. T., Campos, D. P., Barbosa, M. T. S., \& Shimakura, S. E. (2019). Análise de sobrevivência: Teoria e aplicações em saúde (2a ed.). Rio de Janeiro: Fiocruz.

Dallacort, R., Martins, J. A., Inoue, M. H., Freitas, P. S. L., \& Coletti, A. J. (2011). Distribuição das chuvas no município de Tangará da Serra, médio norte do Estado de Mato Grosso, Brasil. Acta Scientiarumtmospheric, 33(2), 193-200. 
Fundação Amazônia de Amparo a Estudo e Pesquisas (FAPESPA). (2016). Estatísticas Municipais Paraenses: Acará. / Diretoria de Estatística e de Tecnologia e Gestão da Informação. - Belém.

Instituto Nacional de Pesquisas Espaciais (INPE)/ Centro de Previsão de Tempo e Estudos Climáticos (CPTEC). (2016) El Niño e La Niña. http://enos.cptec.inpe.br

Instituto Brasileiro de Geografia e Estatística (IBGE). (s/d). Acará Pará - PA Histórico. https://biblioteca.ibge.gov.br/visualizacao/dtbs/para/acara.pdf.

Instituto Brasileiro de Geografia e Estatística (IBGE). (s/d). Produção Agrícola Municipal - PAM. https://www.ibge.gov.br/estatisticasnovoportal/economicas/agricultura-epecuaria/9117-producao-agricola-municipal-culturas-temporarias-e-permanentes.html?=\&t=o-que-e.

Instituto Brasileiro de Geografia e Estatística (IBGE). (2017). Agricultura, Pecuária e Abastecimento: Agropecuária puxa o PIB de 2017. http://www.agricultura.gov.br/noticias/agropecuaria-puxa-o-pib-de-2017.

Instituto Brasileiro de Geografia e Estatística (IBGE). (2017). Brasil/ Pará/ Acará. https://cidades.ibge.gov.br/brasil/pa/acara/panorama.

Moraes, B. C., Costa, J. M. N., Costa, A. C. L., \& Costa, M. H. (2005). Variação espacial e temporal da precipitação no estado do Pará. Acta Amazônica, $35(2), 207-214$

Moraes, D., \& Francisco Filho, M. (2018). Contribuição das chuvas do período da tarde em Belém e possíveis relações com a normal climatológica. Revista Brasileira de Climatologia, 14(23), 17-32.

Nobre, C. A. (2008). Mudanças climáticas e o Brasil - Contextualização. Parcerias Estratégicas, 27, 7-17.

Plano Territorial de Desenvolvimento Rural Sustentável (PTDRS). (2010). Território da Cidadania Transamazônica Estado do Pará. Altamira: FVPP, 134p.

Lima, M. A. de, \& Alves, B. J. R. (2008). Vulnerabilidades, impactos e adaptação à mudança do clima no setor agropecuário e solos agrícolas. Parcerias Estratégicas. 13(27), 73-111.

Lima, P. V. (2014). Análise de métodos para detecção de mudanças estruturais em séries temporais climáticas. Dissertação de Mestrado, Universidade Federal do Rio Grande do Norte, Natal, RN, Brasil.

Lira, B. R. P., Lopes, L. N. A., Chaves, J. R. das, Santana, L. R., \& Fernandes, L. L. (2020). Identificação de Homogeneidade, Tendência e Magnitude da Precipitação em Belém (Pará) entre 1968 e 2018. Anuário do Instituto de Geociências - UFRJ, 43(4), 426-439.

Lopes, M. N. G., Souza, E. B. \& Ferreira, D. B. S. (2013). Climatologia regional da precipitação no estado do Pará. Revista Brasileira de Climatologia, 9(12), 84-102.

Minuzzi, R. B. (2010). Tendências na variabilidade climática de Santa Catarina, Brasil. Revista Brasileira de Engenharia Agrícola e Ambiental. 14(12), 12881293.

Moreira, F. S. A., Ferreira, G. R. B., Dias, L. C. \& Vitorino, M. I. (2019). Variabilidade espaço temporal da precipitação na cidade de Belém-PA e sua relação com a incidência de leptospirose. Revista Brasileira de Geografia Física, 12(1), 71-80.

Moura, M. N., \& Vitorino, M. I. (2012). Variabilidade da precipitação em tempo e espaço associada à zona de convergência intertropical. Revista Brasileira de Meteorologia, 27(4):475-483.

Oliveira, P. T., Silva, C. M. S. \& Lima, K. C. (2017). Climatology and trend analysis of extreme precipitation in subregions of Northeast Brazil. Theoretical and Applied Climatology, 130(1-2), 77-90.

Onyutha, C., Tabari, H., Taye, M. T., Nyandwaro, G. N., \& Willems, P. (2016). Analyses of rainfall trends in the Nile River Basin, Journal of Hydroenvironmental Research, 13, 36-51.

Ribeiro, R. E. P., Ávila, P. R., Brito, J. I., Santos, E. G., Leandro, F. S. (2014). Análise da tendência climática nas séries temporais de temperatura e precipitação de Tucuruí-Pará, Revista Brasileira de Geografia Física, 7(5), 798-807.

SAGRI. 1996. Levantamento da realidade agrícola do Estado do Pará. EMATER-PA. Série Documentos. 5. 65p

Sanches, F. O., May, G. C., Silva, R. V., \& Ferreira, R. V. (2016). Chuvas no norte gaúcho: Um estudo sobre a tendência das chuvas e mudanças climáticas, Revista Brasileira de Climatologia, 18(12), 349-360.

Sena, J. P. O., \& Lucena, D. B. (2013). Identificação da tendência de precipitação na microrregião do Cariri Paraibano, Revista Brasileira de Geografia Física, $6(5), 1400-1416$.

Silva, J. S. O. (2006). Produtividade de óleo de palma na cultura do dendê na Amazônia Oriental: influência do clima e do material genético. Dissertação de Mestrado, Universidade Federal de Viçosa, Minas Gerais, MG, Brasil.

Silva, D. F., Prela-Pantano, A., \& Sant'Anna Neto, J., L. (2008). Variabilidade da precipitação e produtividade agrícola na região do médio Paran apanema, SP. Revista Brasileira de Climatologia, 3.

Silva, F. L., Homma, A. K. O., \& Pena, H. W. A. (2011). O cultivo do dendezeiro na Amazônia: Promessa de um novo ciclo econômico na região, Observatório de La Economía Latinoamericana, 158.

Silva, G. M., Teixeira-Gandra, C. F. A., Damé, R. C. F., Klumb, G. B., \& Veber, P. M. (2015). Tendências de séries de precipitação total mensal para localidades do Rio Grande do Sul, Revista Brasileira de Engenharia e Sustentabilidade, 1(2), 13-22. 
Research, Society and Development, v. 10, n. 2, e39310212134, 2021

(CC BY 4.0) | ISSN 2525-3409 | DOI: http://dx.doi.org/10.33448/rsd-v10i2.12134

Silva Junior, J. R. B. da. (2017). A inserção da matriz produtiva do dendê em áreas antropizadas: Contribuição para um ambiente sustentável. Dissertação de Mestrado, Instituto Tecnológico Vale, Belém, PA, Brasil.

Simas, J. P., \& Pereira, C. L. C. (2019). Energia e sustentabilidade: Análise da viabilidade do cultivo de dendê para o desen volvimento regional endógeno da Amazônia, Boletim de Geografia, 37(1), 184-198.

Souza, E. B., Ferreira, D. B. S., Guimarães, J. T. F., Franco, V. S., Azevedo, F. T. M., Moraes, B. C., \& Souza, P. J. O. P. (2017). Padrões climatológicos e tendências da precipitação nos regimes chuvoso e seco na Amazônia Oriental. Revista Brasileira de Climatologia, 13(21), 81-93.

Yue, S., Pilon, P., \& Cavadias, G. (2002). Power of the Mann-Kendall and Spearman's rho tests for detecting monotonic trends in hydrological series, Journal of Hydrology, 259(1-4), 254-271. 